\title{
Shift of the mean magnetic field values: Effect of scatter due to secular variation and errors
}

\author{
Tadahiro Hatakeyama ${ }^{1,2}$ and Masaru Kono ${ }^{2}$ \\ ${ }^{1}$ Department of Earth and Planetary Science, University of Tokyo, 7-3-1 Hongo, Bunkyo-ku, Tokyo 113-0033, Japan \\ ${ }^{2}$ Institute for Study of the Earth's Interior, Okayama University, 827 Yamada, Misasa, Tottori 682-0193, Japan
}

(Received April 12, 2000; Revised August 4, 2000; Accepted September 25, 2000)

\begin{abstract}
Paleomagnetic data are mostly given in the form of field directions (inclinations and declinations) which depend nonlinearly on the model parameters (Gauss coefficients). Because of this nonlinearity, the means of the data are affected not only by the means of the parameters but also by their fluctuations. Defining the mean directions by the Fisher method decreases this effect but does not completely eliminate it. For various mean fields, we evaluate the effect of secular variation on the means of Fisher-averaged directions by the analytical (Taylor expansion to the second order) as well as by the numerical (Monte Carlo) method. It was shown that a significant amount of offset occurs in the field values because of the fluctuation caused by secular variation. In the case of an inclination anomaly, the effect of secular variation as a function of latitude is antisymmetric about the equator, similar to that of the axial octupole term $\left(g_{3}^{0}\right)$. We also show that the measurement errors do not induce biases in the mean field data, provided that they are random and isotropic.
\end{abstract}

\section{Introduction}

In the characterization of the global magnetic field, the use of spherical harmonic analysis and description by Gauss coefficients is the standard as this gives a complete and unique representation of the source-free magnetic field (Langel, 1987). In the case of the paleomagnetic field, similar approaches have been sought for a long time (Benkova et al., 1970; Creer et al., 1973). However, these early efforts were not very satisfactory since they did not take care of the nonuniqueness associated with the inversion of magnetic direction data (Kono, 1976; Proctor and Gubbins, 1990; Hulot et al., 1997).

Modern inversion methods such as stochastic inversion (Jackson, 1979; Gubbins, 1983; Gubbins and Bloxham, 1985) or harmonic splines (Shure et al., 1982) were developed to take care of these problems. These methods apply some physically plausible conditions, so that the solutions are sought within the bounds that such constraints are satisfied. These inversion methods are also powerful in applications where data quality is far from ideal (large errors, poor site distribution, etc.). Gubbins and Kelly (1993), Johnson and Constable $(1995,1997)$ and Kelly and Gubbins (1997) employed inversion methods adapted to the nonlinear direction data. These authors gave time-averaged magnetic fields for the last 5 million years.

Kono et al. (2000a), however, pointed out that not only the means but also the variances of Gauss coefficients affect the means of nonlinear data, and pointed out that these authors did not properly consider this effect. To avoid the complexity of this problem, Kono et al. (2000a) used only paleointensity

Copy right (C) The Society of Geomagnetism and Earth, Planetary and Space Sciences (SGEPSS); The Seismological Society of Japan; The Volcanological Society of Japan; The Geodetic Society of Japan; The Japanese Society for Planetary Sciences. data in their inversion, which are actually linear data, since information about vector directions is also available for them. This approach is easier but not satisfactory as paleointensity data are much less abundant than paleodirection data.

If we consider the abundance of directional data compared to intensity data, the use of nonlinear data is unavoidable for recovering the paleomagnetic field structure. The suggestion by Kono et al. (2000a) is theoretically correct; there are always contributions to the mean of the field values from the fluctuation of the parameters because of the nonlinearity of the data used. However, their discussion and an analytical estimate by Kono (1997a) were based on this effect seen through the simple means of inclinations and declinations. In paleomagnetism, these angles are not usually averaged separately but the mean direction is defined by the method proposed by Fisher (1953), i.e., taking the direction of the sum of the unit vectors representing the direction of individual measurements. This procedure markedly reduces the bias in the mean. So, if the effect of fluctuations is made small enough by taking the Fisher-type means, a reasonable mean field model can still be obtained from the mean direction data assuming one-to-one correspondence between the data and the parameters. To evaluate the relevance of the mean field models thus far proposed, we need a quantitative estimate of the effect of fluctuations on the mean direction in the case of the Fisher averaging procedure.

In this study, we evaluate the effect of scatter on the mean field when calculated by the Fisher method. For this purpose, we take a few typical mean field models and assume that the behavior of the parameters (Gauss coefficients) in a long-term secular variation can be modeled by treating them as normal variates having some averages corresponding to the mean field and certain variances. As a theoretical approach, we employ the accurate second-order Taylor expan- 
sion. We compare these "analytical" results with the numerical ones obtained by generating random models following the assumed distribution of parameters. We also evaluate the effect of errors in measurements of the mean directions and examine whether these effects can be distinguished from the effects of the secular variation.

\section{Taylor Series Expansion of Magnetic Field Data}

We can express the three orthogonal components $(X, Y$ and $Z$ ) of the geomagnetic field using a potential obtained by spherical harmonic analysis (see Appendix A.1). These components depend linearly on the parameters of the field model (Gauss coefficients). Nonlinear quantities, such as direction cosines $(x, y, z)$, field directions ( $D$ and $I)$, and virtual geomagnetic pole (VGP), can be described by a combination of the linear components (summarized in Appendix A.2 and A.3).

\subsection{Expressions for nonlinear data}

In general, a nonlinear element of the magnetic field $A$ (such as inclination or declination) can be expressed by Taylor expansion around a reference model $\boldsymbol{m}_{0}$ (Kono et al., 2000a)

$$
\begin{aligned}
A= & A_{0}+\sum_{j} A_{j} \Delta m_{j}+\frac{1}{2 !} \sum_{j, k} A_{j k} \Delta m_{j} \Delta m_{k} \\
& +\frac{1}{3 !} \sum_{j, k, l} A_{j k l} \Delta m_{j} \Delta m_{k} \Delta m_{l}+\cdots,
\end{aligned}
$$

where $A_{0}$ is the observation corresponding to the reference model,

$$
A_{0}=A\left(\boldsymbol{m}_{0}\right),
$$

and $\boldsymbol{m}$ is a vector composed of the model parameters (Gauss coefficients), which is a sum of the reference model $\boldsymbol{m}_{0}$ and deviation from it

$$
\boldsymbol{m}=\boldsymbol{m}_{0}+\Delta \boldsymbol{m} .
$$

The subindices attached to a field value indicate derivatives with respect to the components of model $\boldsymbol{m}$; i.e., $A_{j}, A_{j k}$ and $A_{j k l}$ are, respectively, the first, second, and third derivatives of $A$

$$
\begin{aligned}
A_{j} & =\frac{\partial A}{\partial m_{j}}, \quad A_{j k}=\frac{\partial^{2} A}{\partial m_{j} \partial m_{k}}, \\
A_{j k l} & =\frac{\partial^{3} A}{\partial m_{j} \partial m_{k} \partial m_{l}}, \cdots .
\end{aligned}
$$

Note that $j, k, l, \ldots$ span the interval $(1, \ldots, J)$, where $J=$ $\ell_{\max }\left(\ell_{\max }+2\right)$ is the number of parameters, so that the suffix 0 is always reserved for representing the value for the reference model. Explicit forms of the first and second derivatives of various magnetic field data which are useful in paleomagnetic analyses are given in Appendix A. From (1), the mean and the variance of some component of the field can be calculated as

$$
\begin{aligned}
\mathrm{E}[A]= & A_{0}+\sum_{j} A_{j} \mathrm{E}\left[\Delta m_{j}\right] \\
& +\frac{1}{2} \sum_{j, k} A_{j k} \mathrm{E}\left[\Delta m_{j} \Delta m_{k}\right]+\cdots,
\end{aligned}
$$

$$
\begin{aligned}
\mathrm{V}[A]= & \mathrm{E}\left[A^{2}\right]-(\mathrm{E}[A])^{2} \\
= & \sum_{j, k} A_{j} A_{k} \mathrm{E}\left[\Delta m_{j} \Delta m_{k}\right] \\
& +\frac{1}{3} \sum_{j, k, l, n} A_{j} A_{k l n} \mathrm{E}\left[\Delta m_{j} \Delta m_{k} \Delta m_{l} \Delta m_{n}\right] \\
& +\cdots
\end{aligned}
$$

Variations in Gauss coefficients in a long-term secular variation can be modeled by assuming that each $m_{j}$ is an independent random variable following a normal distribution with the mean $\mu_{j}$ and the variance $\sigma_{j}^{2}$ (Constable and Parker, 1988)

$$
m_{j} \sim N\left(\mu_{j}, \sigma_{j}^{2}\right)
$$

If the reference model $\boldsymbol{m}_{0}$ is equated with the mean field model $\boldsymbol{\mu}=\left\{\mu_{j}\right\}$, each $\Delta m_{j}$ becomes a zero-mean normal variate

$$
\Delta m_{j} \sim N\left(0, \sigma_{j}^{2}\right)
$$

Therefore, (5) and (6) are now

$$
\begin{aligned}
\mathrm{E}[A]= & A_{0}+\frac{1}{2} \sum_{j} A_{j j} \sigma_{j}^{2} \\
& +\frac{1}{8} \sum_{j, k} A_{j j k k} \sigma_{j}^{2} \sigma_{k}^{2}+\cdots, \\
\mathrm{V}[A]= & \sum_{j} A_{j}^{2} \sigma_{j}^{2}+\sum_{j, k} A_{j} A_{j k k} \sigma_{j}^{2} \sigma_{k}^{2}+\cdots
\end{aligned}
$$

As we have assumed normal distribution for each $m_{j}$, the means and variances of data depend only on even-order derivatives, so that (9) and (10) are valid to the fourth order, and the next terms are sixth order in $\Delta m_{j}$. In (9), the first term is due to the mean of the model parameters, while the second and third terms show the effects of the variances of the secular variation model on the mean field. If we deal only with linear observations $(X, Y$ and $Z$ ), these terms do not matter because the second and higher-order derivatives of them are all exactly zero. The above equations indicate that the means of nonlinear data $(D, I, \ldots)$ depend not only on the means but are also influenced by the variances of the model parameters (Kono et al., 2000a). This fact complicates the derivation of a time-averaged field from time-averaged data. It may also affect the stability of the iteration procedures when we solve a nonlinear inverse problem.

In the following analyses, we use the equation of the mean (9) truncated at the second order (i.e., up to the terms with $\sigma_{j}^{2}$ ) as the "analytical" expression for the nonlinear quantities.

\subsection{Expressions for the Fisherian means}

The above method can be used to obtain averages of inclination and declination, separately, but the so-called "mean direction" in paleomagnetism is almost always defined by using the method proposed by Fisher (1953). In this procedure, each directional datum is converted to direction cosines

$$
\begin{aligned}
& x_{n}=\cos I_{n} \cos D_{n}, \quad y_{n}=\cos I_{n} \sin D_{n}, \\
& z_{n}=\sin I_{n},
\end{aligned}
$$


and the best estimate of the mean direction is calculated from the $N$ unit vectors as

$$
\bar{x}_{F}=\frac{\sum_{n=1}^{N} x_{n}}{R}, \quad \bar{y}_{F}=\frac{\sum_{n=1}^{N} y_{n}}{R}, \quad \bar{z}_{F}=\frac{\sum_{n=1}^{N} z_{n}}{R},
$$

where $R$ is the length of the sum of $N$ unit vectors,

$$
R=\sqrt{\left(\sum_{n=1}^{N} x_{n}\right)^{2}+\left(\sum_{n=1}^{N} y_{n}\right)^{2}+\left(\sum_{n=1}^{N} z_{n}\right)^{2}} .
$$

By dividing both the numerator and denominator of (12) by $N$, we see that, for instance, $\bar{x}_{F}=E_{x} / E_{f}$, where $E_{x}=$ $\mathrm{E}[x]$, etc., and $E_{f}=\sqrt{E_{x}^{2}+E_{y}^{2}+E_{z}^{2}}$ is the mean displacement in an $N$-step random walk. The mean direction in the sense of Fisher $\left(\bar{D}_{F}\right.$ and $\left.\bar{I}_{F}\right)$ is calculated from these as

$$
\begin{aligned}
& \bar{D}_{F}=\tan ^{-1}\left(\frac{\bar{y}_{F}}{\bar{x}_{F}}\right)=\tan ^{-1}\left(\frac{E_{y}}{E_{x}}\right), \\
& \bar{I}_{F}=\tan ^{-1}\left(\frac{\bar{z}_{F}}{\sqrt{\bar{x}_{F}^{2}+\bar{y}_{F}^{2}}}\right)=\tan ^{-1}\left(\frac{E_{z}}{E_{h}}\right),
\end{aligned}
$$

where $E_{h}=\sqrt{E_{x}^{2}+E_{y}^{2}}$. Thus, it is clear that we need a scheme of Taylor expansion applicable to the above form.

In general, a composite function of $\boldsymbol{m}$

$$
C(\boldsymbol{A})=C\left(A^{(1)}(\boldsymbol{m}), A^{(2)}(\boldsymbol{m}), \cdots\right),
$$

where each $A^{(p)}$ is a function of $\boldsymbol{m}$, can be expanded by the functions $A^{(p)}$

$$
\begin{aligned}
C(\boldsymbol{A})= & C\left(\boldsymbol{A}_{0}\right)+\sum_{p} \frac{\partial C}{\partial A^{(p)}} \Delta A^{(p)} \\
& +\frac{1}{2 !} \sum_{p, q} \frac{\partial^{2} C}{\partial A^{(p)} \partial A^{(q)}} \Delta A^{(p)} \Delta A^{(q)}+\cdots,
\end{aligned}
$$

provided that the intermediate functions $A^{(p)}$ are independent of each other. The expansion (16) is again carried out for the reference model $\boldsymbol{m}_{0}$, and the value of $A^{(p)}(\boldsymbol{m})$ is divided into the part corresponding to the reference model and to the residual

$$
A^{(p)}=A_{0}^{(p)}+\Delta A^{(p)} .
$$

Note that the residual part $\Delta A^{(p)}$ not only contains the first order but also all the higher-order terms in $\Delta m_{j}$ as shown in (1). Equation (16) may be further reduced by Eq. (1). Here, we consider the case where the intermediate functions are the means of the direction cosines

$$
\boldsymbol{A}=\left(E_{x}, E_{y}, E_{z}\right) .
$$

The expansions for these intermediate functions were obtained from Eq. (9). Because the lowest-order term in $\Delta A^{(p)}$ is proportional to $\sigma_{j}^{2}$ (i.e., no first-order term), the expansion form, accurate up to the second order, is simply

$$
\bar{C}_{F}=C_{0}+\frac{1}{2} \sum_{j}\left(\sum_{p} \frac{\partial C}{\partial A^{(p)}} A_{j j}^{(p)}\right) \sigma_{j}^{2}+\cdots .
$$

With this form, we calculate the effect of the geomagnetic secular variation (in Section 3) and the measurement errors (in Section 4) in the Fisher-mean directions. The explicit forms are listed in Appendix A.4.

\section{Effect of Secular Variation on the Mean Field Values}

With the appropriate Taylor expansion forms, we can analytically evaluate the effect of scatter in the data on the mean values of field directions and direction cosines. The scatter in the data are caused either by secular variation of the magnetic field or by errors in various phases of the treatments used to recover the field directions. We will consider only the effect of secular variation in this section and treat the errors in the next section.

Analytical estimation is based on the Taylor series expansion up to second order, using the Fisher-averaging procedure. To supplement the analytical estimates, numerical calculations were done with a $10^{5}$ set of randomly generated Gauss coefficients. This number seems to be enough to satisfactorily represent the distribution of the assumed secular variation. Comparison between these two calculations gives estimates of the effects due to the fourth and higher-order terms.

We consider two types of mean field models; the geocentric axial dipole (GAD, in Subsection 3.2) field, and two nonaxisymmetric field models (in Subsection 3.3). In each case, the variances of Gauss coefficients are assumed to depend only on the degree of the spherical harmonic and not on the order. In a few cases, some of the non-dipole terms were allowed to deviate from the general trend (in Subsection 3.4). Because the IGRF 1995 (Barton, 1997) was used as one of the non-axisymmetric mean fields, all the models were calculated up to the maximum degree of $10\left(\ell_{\max }=10\right)$.

\subsection{Model for secular variation}

Paleomagnetic secular variation (PSV) models have been proposed by various authors (e.g. Cox, 1970; McFadden et al., 1988). In this paper, we adopt a model similar to those proposed by Constable and Parker (1988) and by Kono and Tanaka (1995). These models are based on the observed exponential decay of the power of the geomagnetic field with a spherical harmonic degree. For the present field, an almost flat spectrum is observed at the core-mantle boundary (CMB) (Langel and Estes, 1982). This feature is also seen in the selfconsistent three-dimensional geodynamo simulation models (e.g. Glatzmaier and Roberts, 1995; Kono et al., 2000b).

In the model of Constable and Parker (1988), the power in a degree is divided equally into harmonics of different orders, so that the variances of a degree $\ell$ Gauss coefficient are given as

$$
\sigma_{\ell}^{2}=\frac{(c / a)^{2 \ell}}{(\ell+1)(2 \ell+1)} \alpha^{2},
$$

where $a$ and $c$ are the radii of the Earth and its core, respectively. Constable and Parker (1988) estimated the value of $\alpha$ for the present field as $27.7 \mu \mathrm{T}$, excluding the dipole $(\ell=1)$ terms. A few other terms were permitted to behave differently in the model of Constable and Johnson (1999).

In this paper, we use Eq. (20) with various values of $\alpha$ to define the variances of Gauss coefficients, including the dipole terms. In paleosecular PSV models, we implicitly assume that the statistical properties of the field models are well sampled by observations for a long period of time. In the numerical calculations, we use $10^{5}$ random models to generate "instantaneous observations" of the field at various times, which should represent well the entire distribution of 

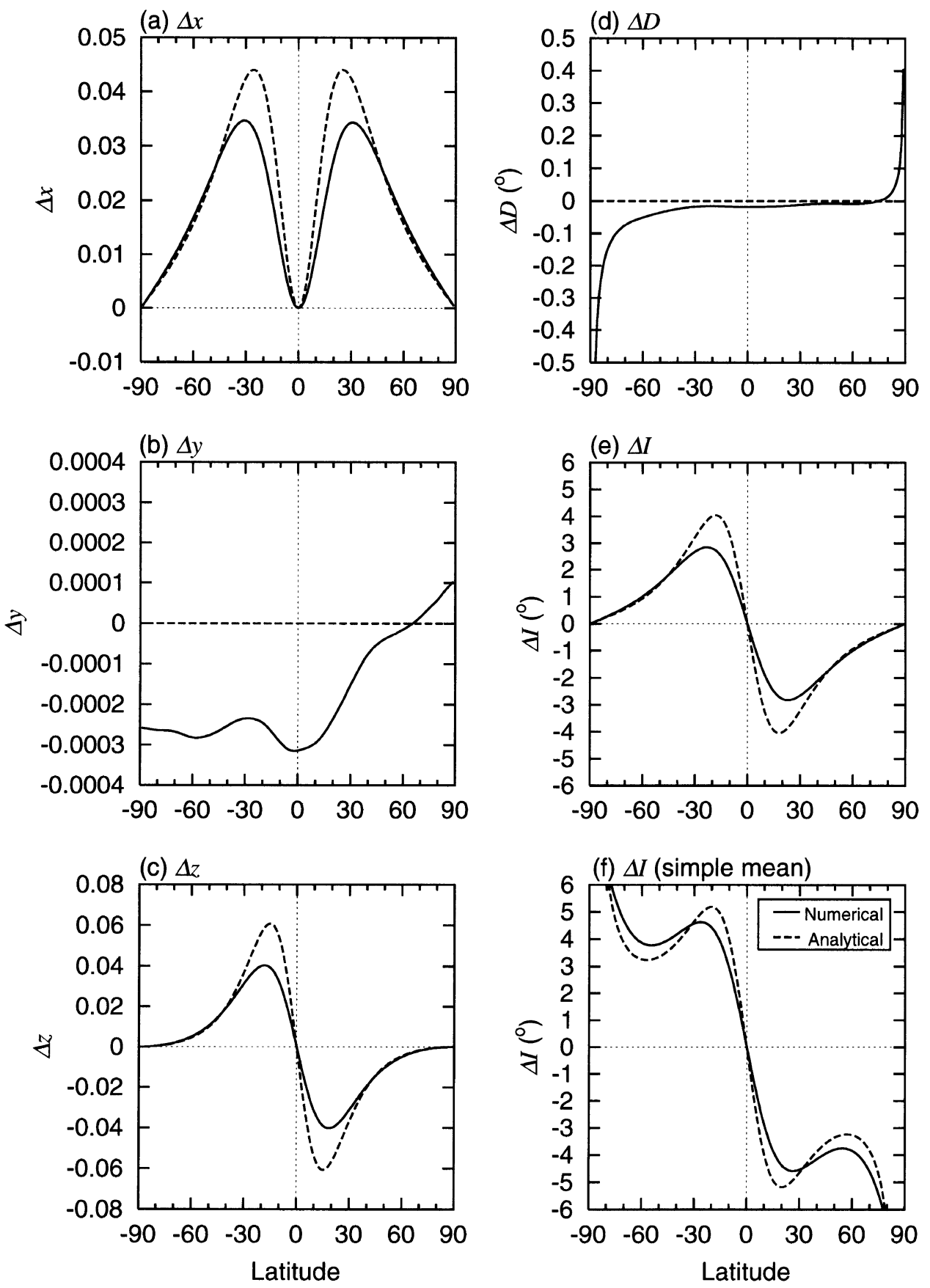

Fig. 1. Latitude dependency of the biases; (a) $\Delta x$, (b) $\Delta y$, (c) $\Delta z$, (d) $\Delta D$ and (e) $\Delta I$; by numerical (solid lines) and second-order analytical (dashed) calculation and (f) $\Delta I$ calculated by a simple averaging method (like in Kono, 1997a). The mean field is due to GAD ( $\left.g_{1}^{0}=-30.0 \mu \mathrm{T}\right)$ and a PSV model $\left(\alpha=30.0 \mu \mathrm{T}\right.$ in Eq. (20)). Note that the values of $y$ and $D$ due to the random data set $\left(N=10^{5}\right)$ are very small and their analytical value is exactly zero.

possible ranges of fluctuation.

\subsection{Fluctuations about the geocentric axial dipole field}

As an example of the simplest model, the GAD field $\left(g_{1}^{0}=-30 \mu \mathrm{T}\right)$ was taken as the mean field. In this case, the fluctuations around the mean with the form (20) produce only an axisymmetric effect. Figure 1 shows the deviations of the means of direction cosines $x, y, z$ and angles $D$ and $I$ from the axial dipole field values. Biases estimated analytically (by Taylor expansion) and numerically (by $10^{5}$ random models) are shown. For Figs. 1(a)-(e), the mean values were determined by the Fisher (1953) method, i.e., the direction of the sum of unit vectors. Figure 1(f), on the other hand, gives the deviation of the simple mean of the inclination from the axial dipole value.

It can be seen that there are indeed biases due to the presence of fluctuations (such as secular variation), except for $D$ and $y$, for which the effect is negligible. These biases take the maxima at a low latitude. For instance, the inclination offset (Fig. 1(e)) for the variation of parameters with $\alpha=30.0 \mu \mathrm{T}$ is $2.8^{\circ}$ at the latitude of $20^{\circ} \mathrm{N}$ or $\mathrm{S}$, and vanishes at the poles or 


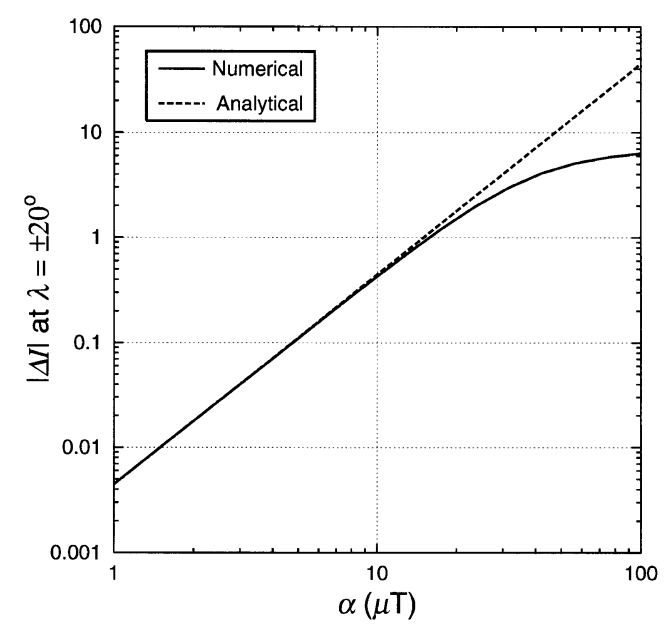

Fig. 2. $\alpha$ dependency of $|\Delta I|$ at the latitude of $20^{\circ} \mathrm{N}$ and $\mathrm{S}$ with the same parameters in Fig. 1, suggesting that the second-order accurate expression (A.24, dashed line) of $|\Delta I|$ always overestimates the numerical value (solid).

on the equator. By simple averaging (Fig. 1(f)) the values of $\Delta I$ are significantly higher than those of the Fisherian mean (Fig. 1(e)). Figure 1(f) also shows that a simple mean makes $\Delta I$ much larger near the poles because of the singularity in its definition (Kono, 1997a).

The inclination differences in the case of the GAD mean field have a trend that the absolute value of inclination becomes smaller everywhere on the Earth's surface. The difference becomes larger with an increase in amplitude $(\alpha)$ of the secular variation. It is only about $0.5^{\circ}$ when $\alpha=10 \mu \mathrm{T}$ (Fig. 2), which can easily be buried by the usual errors in paleomagnetic data. The bias becomes significant for larger $\alpha$ $\left(1.5^{\circ}\right.$ for $20 \mu \mathrm{T}$ and $4^{\circ}$ for $\left.40 \mu \mathrm{T}\right)$. We can conclude that the scatter of data due to secular variation will affect the mean values of observed inclinations at these latitudes if $\alpha$ is $20 \mu \mathrm{T}$ or larger.

The Taylor expansion to the second order (A.24) gives the correct magnitude of inclination bias up to about $\alpha=20$ $\mu \mathrm{T}$. For larger values of $\alpha$, the analytical method gives larger estimates than those obtained by random numerical models. In $\Delta x, \Delta z, \Delta I$ (Fisher means), analytical values always produce overestimates, while $\Delta I$, based on a simple average, is larger or smaller than the numerical estimate depending on the latitude. The difference between the two estimates are about $40 \%$ or less when $\alpha=30 \mu \mathrm{T}$ (Fig. 2).

\subsection{Fluctuation about non-axisymmetric mean fields}

Effects of the secular variation are also examined for two non-axisymmetric mean fields; IGRF 1995 (Barton, 1997) and the time-averaged normal-polarity field model for the last 5 million years (JC97N) given by Johnson and Constable (1997). The IGRF 1995 model actually shows an instantaneous field containing both the mean and the fluctuation parts, but we use it as an example of a field with large non-dipole components compared to JC97N in which the axial dipole component is much more dominant.

Declination is not a well-defined quantity at very high latitudes $\left(|I| \sim 90^{\circ}\right)$, because the expression for $D$ becomes singular at the poles (see Kono, 1997a, b). In reality, $|\Delta D|$ is

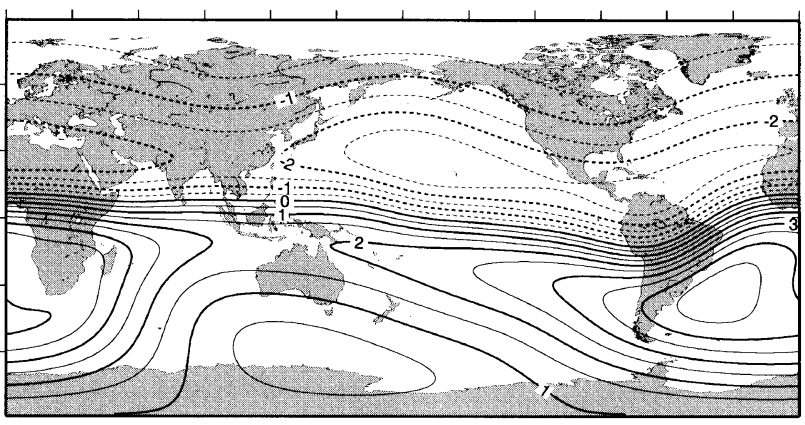

(a) Numerical $\Delta \mathrm{I}$

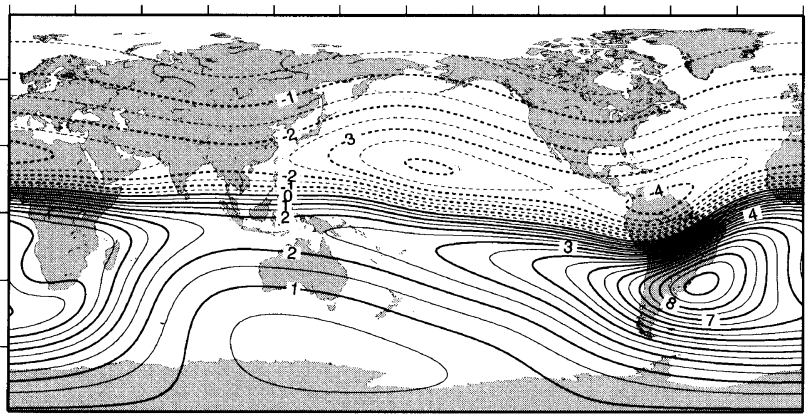

(b) Analytical $\Delta \mathrm{I}$

Fig. 3. Mapping of numerical (a) and second-order analytical (b) $\Delta I$ with the IGRF 1995 mean field and a PSV model of (20) with $\alpha=30.0 \mu \mathrm{T}$.

less than $0.5^{\circ}$ almost everywhere except in the close vicinity of the poles (cf. Fig. 1(d)). Here we only show the maps of inclination offset $\Delta I$ for the mean fields of IGRF 1995 (Fig. 3) and JC97N (Fig. 4) with a variation of $\alpha=30.0 \mu \mathrm{T}$.

In both cases, the contour on which inclination offset vanishes $\left(\Delta I=0^{\circ}\right)$ almost coincides with the geomagnetic equator and $\Delta I$ is negative (positive) when the value of $I$ is positive (negative). Therefore, it can be concluded that the geomagnetic secular variation makes the absolute value of inclination smaller almost everywhere on the Earth's surface (see also Fig. 1(e)). Furthermore, the features of the magnetic field derived from second-order analytic results is very similar to those obtained by numerical calculations. The analytical expression overestimates the bias in a similar way to the case of the GAD mean field. The difference between analytical and numerical estimates becomes conspicuous at sites where the absolute value of inclination is more than $40^{\circ}$ $\left(|\Delta I| \simeq 2^{\circ}\right)$.

In the case of the IGRF 1995 mean field (Fig. 3), inclination bias is particularly large in the south Atlantic. There, the differences between the analytical and numerical estimates are the largest. This is known to be a region in which the effect of the non-dipole field is significant and also a region where the VGPs may have a metastable state during reversals (Hoffman, 1992). Hoffman showed four flux centers and suggested that transitional VGPs cluster in these areas. However, large $\Delta I$ does not seem to be associated with the other three regions (north America, east Indian Ocean and central Asia), possibly because the latter are at high geomagnetic latitudes. 


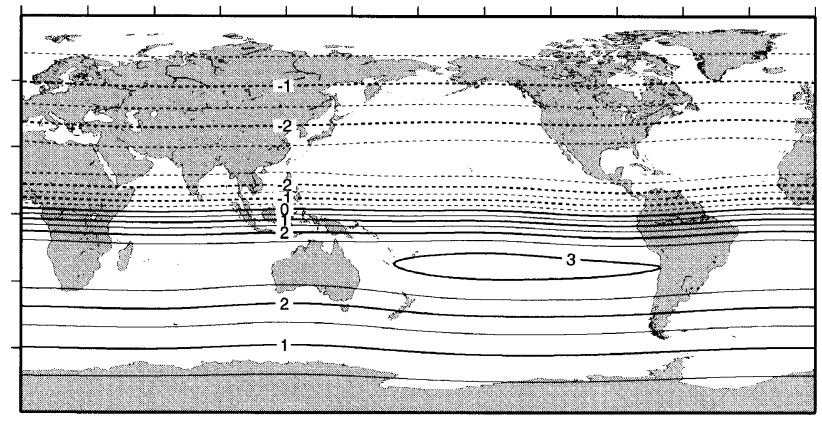

(a) Numerical $\Delta \mathrm{I}$

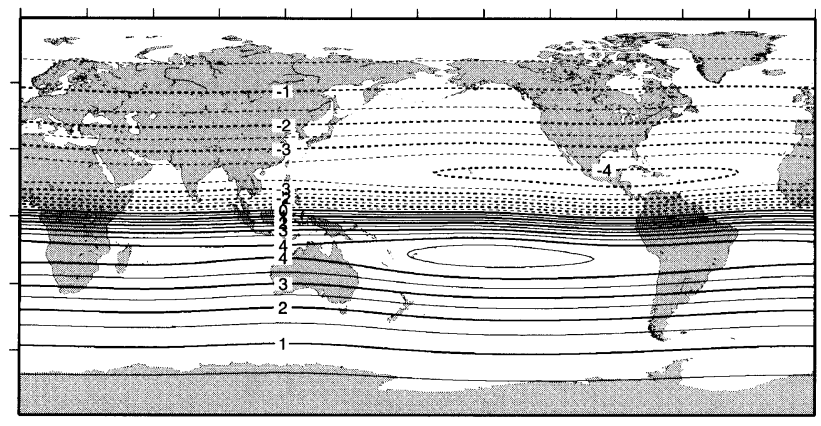

(b) Analytical $\Delta \mathrm{I}$

Fig. 4. Mapping of numerical (a) and second-order analytical (b) $\Delta I$. The mean field is the result of Johnson and Constable (1997) from paleomagnetic data in the normal periods for the last $5 \mathrm{Ma}$ as the mean field, and the PSV model is the same as in Fig. 3.

\subsection{Effect of $(2,1)$ harmonics}

From the latitude dependence of scatter of the field or the VGP components, Kono and Tanaka (1995) and Kono $(1997 \mathrm{a}, \mathrm{b})$ concluded that the variation of the quadrupolar terms $g_{2}^{1}$ and $h_{2}^{1}$ should be a few sizes larger in amplitude than the general trend given by (20). We evaluate the effect of large $(2,1)$ terms on the mean inclinations. Figure 5 shows the contour map of $\Delta I$ corresponding to a model which is the same as the axially symmetric model shown in Fig. 1, except that the standard deviation of $g_{2}^{1}$ is made three times larger, as is given by (20). Four (positive and negative) extrema are shown in the $\Delta I$ map, which correspond to the flux patches of the $Z$ field due to $g_{2}^{1}$.

Figure 6 shows the map of $\Delta I$ with a secular variation model in which both of the $(2,1)$ harmonics have larger amplitudes of fluctuation; i.e., $\sigma_{g_{2}^{1}}=\sigma_{h_{2}^{1}}=3 \sigma_{2}$. In this case, the effect becomes axisymmetric because the sum of $g_{2}^{1}$ and $h_{2}^{1}$ contributions is a constant of longitude (Kono, 1997b). The total amount of secular variation is larger in this model compared to 3.2 , so that the $\Delta I$ is much larger. The latitudinal plot (Fig. 6(c)) indicates that the maximum of inclination bias appears at a latitude (about $30^{\circ}$ ) which is higher than in the original PSV model (about $20^{\circ}$, Fig. 1(e)). The reason for this shift seems to be because the field due to a combination of $g_{2}^{1}$ and $h_{2}^{1}$ has nodes at the north and south poles and at the equator. Therefore, the effect of the secular variation by enhanced $g_{2}^{1}$ and $h_{2}^{1}$ is large at middle latitude which is higher than the peak by a PSV model following Eq. (20).

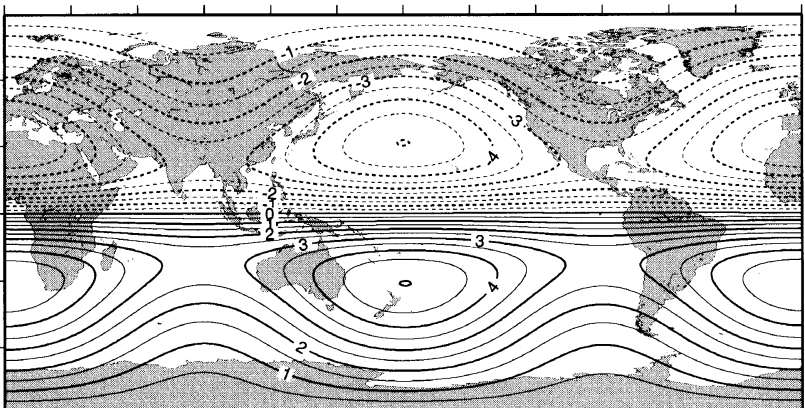

(a) Numerical $\Delta \mathrm{I}$

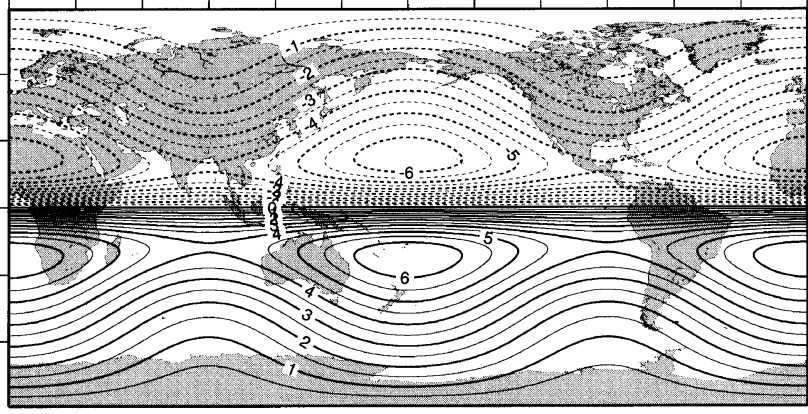

(b) Analytical $\Delta \mathrm{I}$

Fig. 5. Mapping of numerical (a) and second-order analytical (b) $\Delta I$ with the mean field of GAD $\left(g_{1}^{0}=30.0 \mu \mathrm{T}\right)$ and variances from a secular variation model following (20) with $\alpha=30 \mu \mathrm{T}$ excluding $\sigma_{g_{2}^{1}}=3 \sigma_{2}$.

\section{Effects of Measurement Errors on the Mean Di- rection}

In paleomagnetic studies, the data are inevitably contaminated by some amount of experimental errors, even if we collect and measure the samples very carefully. These errors are due to many causes; orientation errors during sampling and cutting, shape anisotropy of samples, heterogeneity of magnetization in a specimen, errors of setting in the magnetometer, errors in the measurements, and so on. Here we omit systematic errors (such as is caused by a strong local magnetic anomaly at sampling sites, or the inability to restore the original bedding plane), and only consider random errors in each measurement.

By excluding the possibility of systematic errors, we can assume that the errors of the measured components $X, Y$ and $Z$ are mutually independent and zero-mean normal variates. It seems reasonable to assume that the standard deviations in individual components are proportional to its intensity

$$
\sigma_{X}=\sigma_{Y}=\sigma_{Z}=s F,
$$

where $s$ is a constant. (This assumption, in effect, is based on the experience that the source of the largest errors in paleomagnetism of volcanic rocks is the orientation errors.)

In the expansion of the means defined by the Fisher procedure (A.24), all the second-order terms (A.26) vanish. Thus, the relevant forms of expansion are

$$
\bar{x}_{F}=\frac{X}{F}+\mathcal{O}\left(s^{4}\right), \quad \bar{y}_{F}=\frac{Y}{F}+\mathcal{O}\left(s^{4}\right),
$$




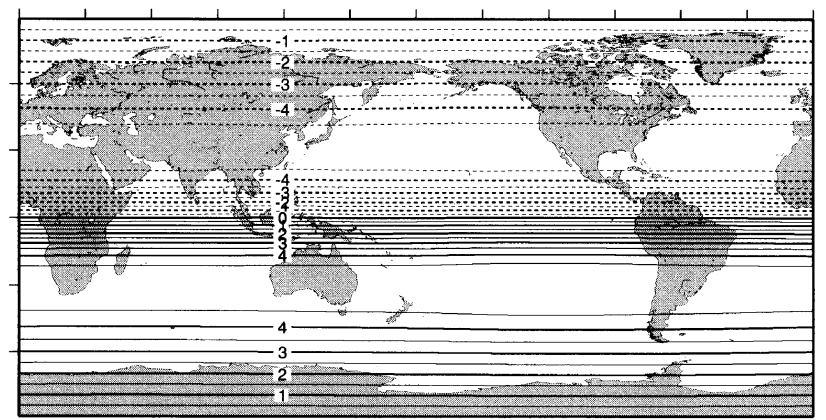

(a) Numerical $\Delta \mathrm{I}$

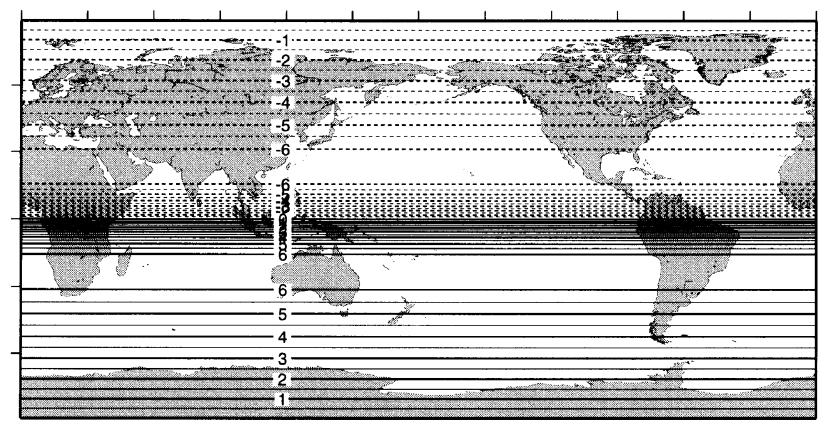

(b) Analytical $\Delta \mathrm{I}$

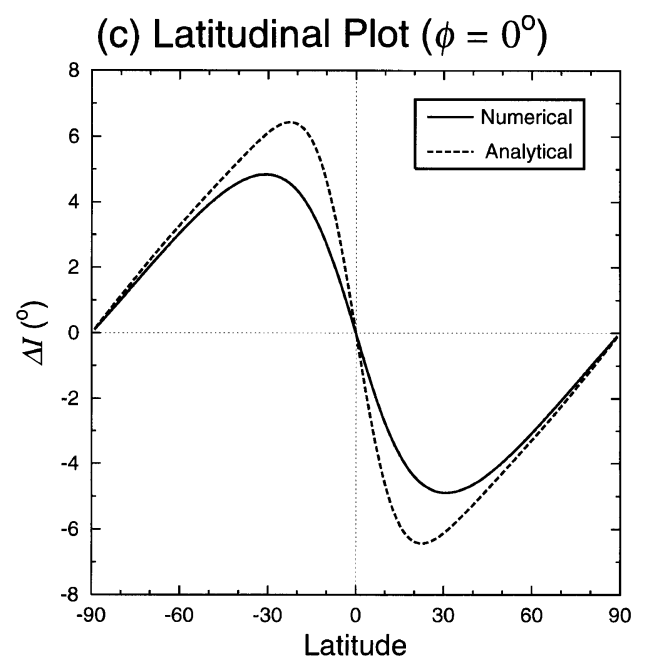

Fig. 6. Mapping of numerical (a) and second-order analytical (b) $\Delta I$, and their sectoral section at $\phi=0^{\circ}$ (c). The mean and variances of Gauss coefficients are the same as in Fig. 5 excluding $h_{2}^{1}$ as well as $g_{2}^{1}$ $\left(\sigma_{g_{2}^{1}}=\sigma_{h_{2}^{1}}=3 \sigma_{2}\right)$.

$$
\begin{aligned}
& \bar{z}_{F}=\frac{Z}{F}+\mathcal{O}\left(s^{4}\right), \quad \bar{D}_{F}=\tan ^{-1} \frac{Y}{X}+\mathcal{O}\left(s^{4}\right), \\
& \bar{I}_{F}=\tan ^{-1} \frac{Z}{\sqrt{X^{2}+Y^{2}}}+\mathcal{O}\left(s^{4}\right) .
\end{aligned}
$$

These indicate that the means of the data are not affected by the presence of scatter due to experimental errors, at least to this order.

To show that these analytical conclusions are correct, deviations from the mean field values $(\Delta D, \Delta I, \Delta x, \Delta y$ and $\Delta z)$ were obtained by the random data set $\left(N=10^{5}\right)$, and the results are shown in Fig. 7 in which the mean field is GAD and $s=0.1$ ( $10 \%$ of the total intensity, which is large for usual experimental situations). As can be seen from this figure, the difference in the means is negligibly small which is in agreement with the above analysis. The small differences shown in this figure are likely to be caused by the incompleteness of the random models, because five different random data sets gave quite different results. From these, we can conclude that if we make adequate observations at each site, there will be no bias to the mean direction due to the experimental errors, provided that the errors are not systematic. Note that this conclusion applies only to the Fisherian means; a significant amount of shallowing results from the scatter due to errors if a simple mean of inclination is taken (e.g., Kono, 1997a). Observational errors also increase the scatter in the data compared to the case with only secular variation.

It is remarkable that the (non-systematic) measurement errors do not cause biases in the means of the field directions. This is quite different from the behavior of the scatter caused by secular variation. In retrospect, it is quite reasonable that the experimental errors do not induce bias in the mean field direction, since the Fisher method was created to obtain the most likely estimate of the magnetization direction when data contain random errors (Fisher, 1953). This feature, however, does not apply to secular variation because it generates nonrandom scatter.

\section{Discussion}

\subsection{Far side effect}

Previous paleomagnetic studies (Wilson, 1970, 1971) suggested that observed inclination is shallower than that produced by the GAD field everywhere in the world. This effect is most prominently seen in the Northern Hemisphere. The VGPs for these data are located on the far side of the geographic North Pole. For example, the lava data set from Hawaii indicates that the inclination is $9^{\circ}$ shallower than the GAD, resulting in an offset of $5^{\circ}$ of the mean VGP from the North Pole (Tanaka, 1999). Combining a small amount of Southern Hemisphere data, Wilson (1970) concluded that this effect can be attributed to the offset of the axial dipole from the geocenter. Equivalently, the "shallow inclination" or "far side effect" can also be explained by the existence of a non-zero axial quadrupole field $\left(g_{2}^{0}\right)$ which has the same sign as that of $g_{1}^{0}$.

With the addition of more data from the Southern Hemisphere, however, it became apparent that the addition of the quadrupole term cannot completely explain the inclination anomalies. Indeed, McElhinny et al. (1996) showed that the inclination anomaly with respect to the GAD value has an asymmetric component. They explained this by introducing the non-zero octupole term $\left(g_{3}^{0}\right)$, in addition to the dipole and quadrupole in the time-averaged field, as the inclination anomaly caused by $g_{2}^{0}$ is symmetric, while that caused by $g_{3}^{0}$ is antisymmetric about the Equator. We have shown, however, that the effect of random secular variation is similar to that of persistent $g_{3}^{0}$ in creating an inclination anomaly antisymmetric about the Equator (Fig. 1(e)). Thus, the inclination anomaly observed for the last $5 \mathrm{Ma}$ (McElhinny et al., 1996) can be equally explained by the combination of 

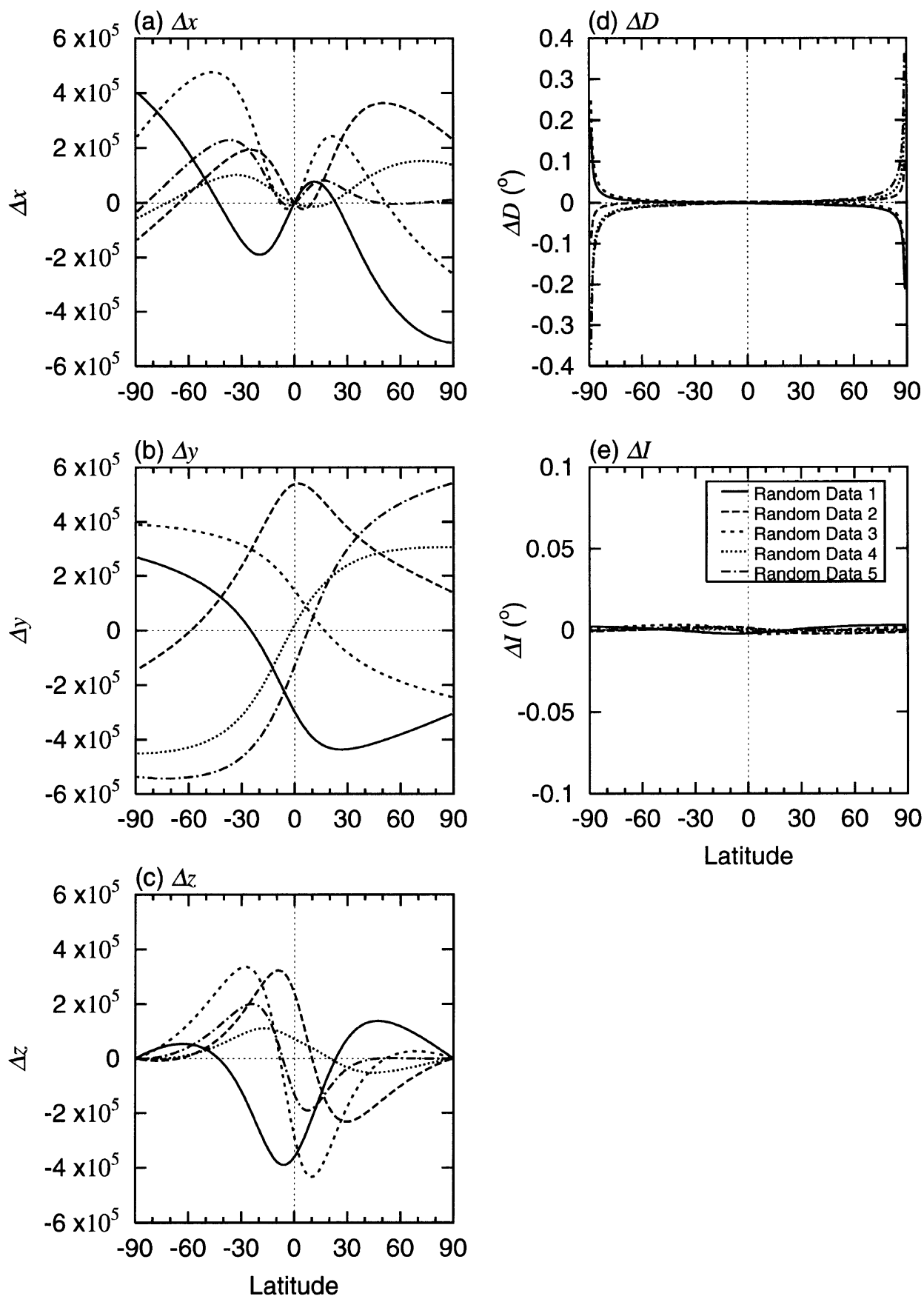

Fig. 7. (a) $\Delta x$, (b) $\Delta y$, (c) $\Delta z$, (d) $\Delta D$ and (e) $\Delta I$ by measurement error with $s=0.1$. There are 5 curves by $10^{5}$ random normal distribution data sets generated by different seeds. They indicate that the amount of random data $\left(N=10^{5}\right)$ is not enough to see the effect of the measurement errors and, even if it exists, it is much smaller than these shown values.

$g_{1}^{0}+g_{2}^{0}+g_{3}^{0}$ (McElhinny et al., 1996) or by $g_{1}^{0}+g_{2}^{0}$ combined with the effect of secular variation (Fig. 8). In the present case, it is not possible to choose the most probable model from the fit shown in Fig. 8 alone. But it may be important that the observed asymmetry does not necessarily mean the existence of the octupole term.

\subsection{Effects of the secular variation and measurement errors on nonlinear inversion}

In this study, we considered the effects of variances due to two sources; geomagnetic secular variation and measurement error. From the foregoing analyses, it can be concluded that secular variation really influences the mean directions, but the errors do not change the means. The effect of the geomagnetic secular variation is to make the absolute value of observed inclination smaller everywhere.

The effect of secular variation was not considered in previous nonlinear inversion studies (Gubbins and Kelly, 1993; Kelly and Gubbins, 1997; Johnson and Constable, 1995, 1997) which sought Gauss coefficients corresponding to the time-averaged field. The present study indicated, however, that the fluctuations of the model parameters caused by secular variation can change the mean value of the observed direc- 


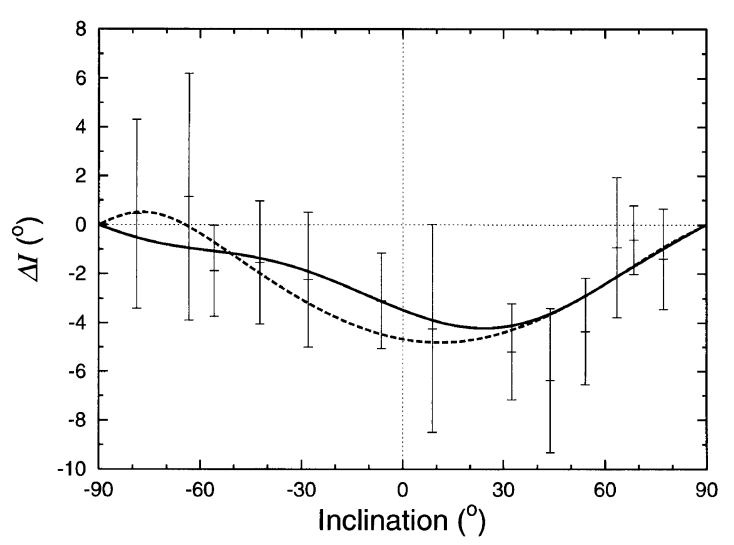

Fig. 8. Inclination anomaly $\Delta I$ for the normal polarity data (the mean and the $95 \%$ confidence interval) of the last $5 \mathrm{Ma}$ (McElhinny et al., 1996). Dashed curve shows their best estimate with $g_{2}^{0} / g_{1}^{0}=0.043$, $g_{3}^{0} / g_{1}^{0}=0.017, g_{4}^{0} / g_{1}^{0}=-0.008$. Solid curve indicates an example of the fit with $g_{2}^{0} / g_{1}^{0}$ plus the effect of secular variation expressed by $\alpha=0.6\left|g_{1}^{0}\right|$.

tions significantly, even when the mean directions are defined as the best estimate of Fisher (1953). The neglect of this effect will result in the estimates of nondipole components with larger errors. Therefore, it is possible that the time-averaged field may be characterized by parameters somewhat different from those given by their results.

Considering these effects, we can define a new inversion method to seek the time-averaged geomagnetic field and its variances (i.e. PSV) from paleomagnetic data. The mean directions of the observed paleomagnetic data depend on the mean Gauss coefficients as well as on their change due to PSV. The observation equation for the mean direction (or the direction cosines) at a site $i$ can be obtained from Eq. (A.24) as

$$
A_{i}=f_{i}(\boldsymbol{\mu}, \boldsymbol{\sigma})
$$

where we take the means $\left\{\mu_{j}\right\}$ and the variances $\left\{\sigma_{j}\right\}$ of Gauss coefficients as the model parameters to be determined.

In addition, we can define another equation to show the relation between the variances of the parameters and the variances of the observations. The equation for variances (or standard deviations) depends on the means as well as the variances of Gauss coefficients and the measurement errors

$$
V_{i}=g_{i}(\boldsymbol{\mu}, \boldsymbol{\sigma}, \boldsymbol{e}) .
$$

It is necessary to solve (23) and (24) simultaneously in order to properly account for the means and variances of the data. Up to now, such inversion was done only for the linear case (Kono et al., 2000a), but a solution for the nonlinear data set is also required.

\subsection{Robustness of the present results}

We have obtained the expressions which indicate the effect of scatter caused by secular variation of the magnetic field on the mean values of field data. It was shown that the effect was not negligible; indeed a considerable amount of offset was observed in the mean values of inclination and other field elements. However, in order to derive workable formulas, it was necessary to make a few assumptions about the field behavior. Thus, the relevance of the present results in secular variation analysis depends on the validity of the assumptions we employed in deriving the expressions.

We would argue that the assumptions employed are quite reasonable and, moreover, the results do not critically depend on the details of the assumptions. First of all, it is quite certain that there were fluctuations in the field elements with time; there is no doubt that the secular variation of the magnetic field occurred with considerable amplitudes. Secondly, the assumption of normal distribution of Gauss coefficients was used more for the convenience of calculation. The Taylor expansion method developed by Kono and Tanaka (1995) and following papers is quite general and applies to any distributions if the existence of low-degree moments (e.g. the mean and variance) can be assumed. Thirdly, using essentially the same assumptions, Constable and Parker (1988) and others (Kono and Tanaka, 1995; Kono, 1997a, b; Constable and Johnson, 1999) obtained reasonable results in the analysis of paleosecular variation in the last $5 \mathrm{Ma}$, which indicates that it is at least permissible. Lastly, Kono et al. (2000b) showed that numerical dynamo simulation results suggests that the distribution of Gauss coefficients of low degrees are indeed quite similar to normal distribution.

It is impossible at the present stage to convincingly show which distribution is more appropriate to describe the fluctuating Gauss coefficients because of the secular variation. However, it may be reasonable to consider that the distribution of $g_{\ell}^{m}$ or $h_{\ell}^{m}$ is effectively bounded in some interval and that it is not skewed. This makes modeling by normal distribution quite appropriate. Moreover, if the distribution of Gauss coefficients deviates from the normal distribution, the nonlinear effects will be larger than those we derived because the effect of the third (skewness) and fourth moment may not be neglected.

\section{Conclusions}

We have quantitatively evaluated the effect of geomagnetic secular variation and measurement errors on the mean directions. If the measurement errors are not systematic, i.e., truly random and isotopic, they do not affect the mean directions but make the variances larger. The fluctuations in the model due to PSV change the mean directions in which the absolute inclination becomes smaller than that for the mean field. For example, in a PSV model with $\alpha=30.0 \mu \mathrm{T}, \Delta I$ is a few degrees at low to middle latitude sites. In the case where the mean field is the GAD, this inclination anomaly is antisymmetric about the Equator, which is similar to that caused by the non-zero mean value of the axial octupole $\left(g_{3}^{0}\right)$. It is possible that the $g_{3}^{0}$ term reported from the analysis of secular variation data (e.g., McElhinny et al., 1996) is partly attributable to this effect. We cannot ignore this effect when we seek the time-averaged field by nonlinear inversion from paleomagnetic data.

We gave the Taylor series expansion of the mean directions up to the second order, where the mean is defined by Fisher's procedure. These expressions agree quite well with the estimates obtained numerically when the amplitude of secular variation is not too large. Some significant difference was observed for the amplitude which is of interest in the actual paleomagnetic data analysis. However, even when the amplitude of the variation is quite large, the main features 
of the biases due to the PSV are very well reproduced by the analytical method in the distribution over the globe, indicating the usability of the second-order Taylor expansion approximation.

Acknowledgments. We thank Yozo Hamano and Hidefumi Tanaka for useful discussions. We also thank Michael McElhinny and Hideo Tsunakawa for constructive criticism. T. Hatakeyama was supported by a Japan Society for Promotion of Science Research Fellowship for Young Scientists. Part of this study was supported by a Grant-in-Aid for Scientific Researches (No. 10440124).

\section{References}

Barton, C. E., International Geomagnetic Reference Field: The seventh generation, J. Geomag. Geoelectr., 49, 123-148, 1997.

Benkova, N. P., N. V. Adam, and T. N. Cherevko, Application of spherical harmonic analysis to magnetic declination data, Geomagn. Aeron., 10, 673 (Engl. Trans. 527-532), 1970 (in Russian).

Constable, C. G. and C. L. Johnson, Anisotropic paleosecular variation models: implications for geomagnetic field observables, Phys. Earth Planet. Inter., 115, 35-51, 1999.

Constable, C. G. and R. L. Parker, Statistics of the geomagnetic secular variation for the past 5 m.y., J. Geophys. Res., 93, 11569-11581, 1988.

Cox, A. V., Latitude dependence of the angular dispersion of the geomagnetic field, Geophys. J. R. astr. Soc., 20, 253-269, 1970.

Creer, K. M., D. T. Georgi, and W. Lowrie, On the representation of the Quaternary and late tertiary geomagnetic fields in terms of dipoles and quadrupoles, Geophys. J. R. astr. Soc., 33, 323-345, 1973.

Fisher, R. A., Dispersion on a sphere, Proc. R. Soc. Lond., A217, 295-305, 1953.

Glatzmaier, G. A. and P. H. Roberts, A three-dimensional self-consistent computer simulation of a geomagnetic field reversal, Nature, 377, 203209, 1995.

Gubbins, D., Geomagnetic field analysis-I. stochastic inversion, Geophys. J. R. astr. Soc., 73, 641-652, 1983.

Gubbins, D. and J. Bloxham, Geomagnetic field analysis-III. Magnetic fields on the core-mantle boundary, Geophys. J. R. astr. Soc., 80, 695-713, 1985.

Gubbins, D. and P. Kelly, Persistent patterns in the geomagnetic field over the past 2.5 Myr, Nature, 365, 829-832, 1993.

Hoffman, K. A., Dipolar reversal states of the geomagnetic field and coremantle dynamics, Nature, 359, 789-794, 1992.

Hulot, G., A. Khokholov, and J.-L. Le Mouël, Uniqueness of mainly dipolar magnetic fields recovered from directional data, Geophys. J. Int., 129, 347-354, 1997.

Jackson, D. D., The use of a priori data to resolve non-uniqueness in linear inversion, Geophys. J. R. astr. Soc., 57, 137-157, 1979.

Johnson, C. L. and C. G. Constable, The time-averaged geomagnetic field as recorded by lava flows over the past $5 \mathrm{Myr}$, Geophys. J. Int., 122, 488-519, 1995.

Johnson, C. L. and C. G. Constable, The time-averaged geomagnetic field: global and regional biases for 0-5 Ma, Geophys. J. Int., 131, 643-666 1997.

Kelly, P. and D. Gubbins, The geomagnetic field over the past $5 \mathrm{Myr}$, Geophys. J. Int., 128, 315-330, 1997.

Kono, M., Uniqueness problems in their spherical harmonic analysis of the geomagnetic field direction data, J. Geomag. Geoelectr., 28, 11-29, 1976.

Kono, M. Paleosecular variation in field directions due to randomly varying Gauss coefficients, J. Geomag. Geoelectr., 49, 615-631, 1997a.

Kono, M. Distributions of paleomagnetic directions and poles, Phys. Earth Planet. Inter., 103, 313-327, $1997 \mathrm{~b}$.

Kono, M. and O. Hiroi, Paleosecular variation of field intensities and dipole moments, Earth Planet. Sci. Lett., 139, 251-262, 1996.

Kono, M. and H. Tanaka, Mapping the Gauss coefficients to the pole and the models of paleosecular variation, J. Geomag. Geoelectr., 47, 115-130, 1995.

Kono, M., H. Tanaka, and H. Tsunakawa, Spherical harmonic analysis of paleomagnetic data: the case of linear mapping, J. Geophys. Res., 105, 5817-5833, 2000a.

Kono, M., A. Sakuraba, and M. Ishida, Dynamo simulation and paleosecular variation models, Phil. Trans. R. Soc. Lond., A358, 1123-1139, 2000b.

Langel, R. A., The main field, in Geomagnetism, vol. 1, edited by J. A. Jacobs, pp. 249-512, Academic Press, London, 1987.

Langel, R. A. and R. H. Estes, A geomagnetic field spectrum, Geophys. Res. Lett., 9, 250-253, 1982.

McElhinny, M. W., P. L. McFadden, and R. T. Merrill, The time-averaged paleomagnetic field 0-5 Ma, J. Geophys. Res., 101, 25007-25027, 1996

McFadden, P. L., R. T. Merrill, and M. W. McElhinny, Dipole/quadrupole family modeling of paleosecular variation, J. Geophys. Res., 93, 1158311588, 1988.

Proctor, M. R. E. and D. Gubbins, Analysis of geomagnetic directional data, Geophys. J. Int., 100, 69-77, 1990.

Shure, L., R. L. Parker, and G. E. Backus, Harmonic splines for geomagnetic modelling, Phys. Earth Planet. Inter., 28, 215-229, 1982.

Tanaka, H., Circular asymmetry of the paleomagnetic directions observed at low latitude volcanic sites, Earth Planets Space, 51, 1279-1286, 1999

Wilson, R. L., Permanent aspects of the Earth's non-dipole magnetic field over upper tertiary times, Geophys. J. R. astr. Soc., 19, 417-437, 1970.

Wilson, R. L., Dipole offset-The time average palaeomagnetic field over the past 25 million years, Geophys. J. R. astr. Soc., 22, 491-504, 1971.

T. Hatakeyama (e-mail: hatake@sys.eps.s.u-tokyo.ac.jp) and M. Kono 


\section{Appendix A. Derivatives of Various Field Components}

The derivatives of paleomagnetic data with respect to Gauss coefficients have been described by various authors (e.g., Constable and Parker, 1988; Kono and Tanaka, 1995; Kono and Hiroi, 1996; Kono, 1997a, b). However, in many cases, these expressions were given in a form applicable to some specific model (e.g., near GAD), and the notations also vary greatly in each case. For the sake of convenience, we give here the complete form of derivatives of the first and second order.

\section{A.1 The linear components of the geomagnetic field}

The magnetic field outside the Earth's core can be described by a scalar potential. The potential $W$ at a point $(r, \theta, \phi)$, where $r$ is the radius, $\theta$ is the colatitude, and $\phi$ is the longitude, is given by

$$
W=a \sum_{\ell=1}^{\ell_{\max }} \sum_{m=0}^{\ell}\left(\frac{a}{r}\right)^{\ell+1}\left(g_{\ell}^{m} \cos m \phi+h_{\ell}^{m} \sin m \phi\right) P_{\ell}^{m}(\cos \theta)
$$

where $P_{\ell}^{m}$ is the Schmidt-normalized Legendre function, $g_{\ell}^{m}$ and $h_{\ell}^{m}$ are Gauss coefficients, and $\ell_{\max }$ is the maximum degree at which spherical harmonics are truncated. The model $\boldsymbol{m}$ is a vector formed by arranging the Gauss coefficients by degree and order

$$
\boldsymbol{m}=\left(g_{1}^{0}, g_{1}^{1}, h_{1}^{1}, g_{2}^{0}, g_{2}^{1}, h_{2}^{1}, \ldots\right)^{T} .
$$

Its components will be designated by a single suffix $j\left(j=1, \ldots, \ell_{\max }\left(\ell_{\max }+2\right)\right)$, such as $m_{j}$, rather than by $\ell$ and $m$.

The three components of the magnetic field at some point over the surface of the Earth can be derived from the potential as

$$
X=\sum_{j} X_{j} m_{j}, \quad Y=\sum_{j} Y_{j} m_{j}, \quad Z=\sum_{j} Z_{j} m_{j},
$$

where $X_{j}, Y_{j}, Z_{j}$ are functions of position $(\theta, \phi)$

$$
X_{j}=\frac{d P_{\ell}^{m}}{d \theta}\left(\begin{array}{c}
\cos m \phi \\
\sin m \phi
\end{array}\right), \quad Y_{j}=\frac{m P_{\ell}^{m}}{\sin \theta}\left(\begin{array}{c}
\sin m \phi \\
-\cos m \phi
\end{array}\right), \quad Z_{j}=-(\ell+1) P_{\ell}^{m}\left(\begin{array}{c}
\cos m \phi \\
\sin m \phi
\end{array}\right) .
$$

The choice of $\phi$ dependence is determined by whether $m_{j}$ is $g_{\ell}^{m}$ or $h_{\ell}^{m}$. Obviously, the three components $(X, Y, Z)$ are linear in Gauss coefficients, and $X_{j}, Y_{j}, Z_{j}$ are their derivatives with respect to a Gauss coefficient $m_{j}$.

Any other field component, either linear or nonlinear, can be written by a combination of these three components (in some cases, the coordinates of the position $r, \theta, \phi$ are also needed).

\section{A.2 Nonlinear field elements}

In paleomagnetism, data are mostly given by two angles, inclination $I$ and declination $D$. In paleointensity experiments, the total intensity $F$ is determined. These quantities are related to the three components as

$$
I=\tan ^{-1}\left(\frac{Z}{H}\right), \quad D=\tan ^{-1}\left(\frac{Y}{X}\right), \quad F=\sqrt{X^{2}+Y^{2}+Z^{2}},
$$

where $H=\sqrt{X^{2}+Y^{2}}$. The first and second derivatives of these quantities are

$$
\begin{aligned}
\frac{\partial I}{\partial m_{j}}= & \frac{H^{2} Z_{j}-Z\left(X X_{j}+Y Y_{j}\right)}{F^{2} H}, \\
\frac{\partial^{2} I}{\partial m_{j} \partial m_{k}}= & \frac{1}{F^{4} H^{3}}\left[\left(F^{2}+2 H^{2}\right) Z\left(X X_{j}+Y Y_{j}\right)\left(X X_{k}+Y Y_{k}\right)-F^{2} H^{2} Z\left(X_{j} X_{k}+Y_{j} Y_{k}\right)\right. \\
& \left.+H^{2}\left(Z^{2}-H^{2}\right)\left\{\left(X X_{j}+Y Y_{j}\right) Z_{k}+\left(X X_{k}+Y Y_{k}\right) Z_{j}\right\}-2 H^{4} Z Z_{j} Z_{k}\right], \\
\frac{\partial D}{\partial m_{j}}= & \frac{X Y_{j}-Y X_{j}}{X^{2}+Y^{2}}, \\
\frac{\partial^{2} D}{\partial m_{j} \partial m_{k}}= & -\frac{\left(X X_{k}+Y Y_{k}\right)\left(X Y_{j}-Y X_{j}\right)+\left(X X_{j}+Y Y_{j}\right)\left(X Y_{k}-Y X_{k}\right)}{H^{4}}, \\
\frac{\partial F}{\partial m_{j}}= & \frac{G_{j}}{F}, \\
\frac{\partial^{2} F}{\partial m_{j} \partial m_{k}}= & \frac{G_{j k}}{F}-\frac{G_{j} G_{k}}{F^{3}},
\end{aligned}
$$

where $H=\sqrt{X^{2}+Y^{2}}$ is the horizontal component and $G=F^{2} / 2, G_{j}=X X_{j}+Y Y_{j}+Z Z_{j}$, etc.

For direction cosines,

$$
x=\cos I \cos D=\frac{X}{F}, \quad y=\cos I \sin D=\frac{Y}{F}, \quad z=\sin I=\frac{Z}{F},
$$


the first and second derivatives of $x$ are

$$
\begin{aligned}
\frac{\partial x}{\partial m_{j}}= & \frac{X_{j}}{F}-\frac{X G_{j}}{F^{3}}, \\
\frac{\partial^{2} x}{\partial m_{j} \partial m_{k}}= & -\frac{1}{F^{3}}\left(X_{j} G_{k}+X_{k} G_{j}+X G_{j k}\right)+\frac{3 X G_{j} G_{k}}{F^{5}}, \\
\frac{\partial^{3} x}{\partial m_{j} \partial m_{k} \partial m_{l}}= & -\frac{1}{F^{3}}\left(X_{j} G_{k l}+X_{k} G_{j l}+X_{l} G_{j k}\right) \\
& +\frac{3}{F^{5}}\left[X_{j} G_{k} G_{l}+X_{k} G_{j} G_{l}+X_{l} G_{j} G_{k}+X\left(G_{j k} G_{l}+G_{j l} G_{k}+G_{j} G_{k l}\right)\right] \\
& -\frac{15}{F^{7}} X G_{j} G_{k} G_{l} .
\end{aligned}
$$

Derivatives of $y$ and $z$ can be obtained by replacing $X$ by $Y$ or $Z$.

\section{A.3 Virtual geomagnetic pole (VGP) and its moment}

In many cases, VGPs or virtual dipole moments (VDMs) are used in place of field elements because the concept of the dipole is global and suitable for worldwide comparison. The expressions for VGP and VDM have been already reported (Kono and Tanaka, 1995; Kono and Hiroi, 1996; Kono, 1997a). The colatitude $\left(\theta_{p}\right)$ and longitude $\left(\phi_{p}\right)$ of VGP are given by

$$
\theta_{p}=\cos ^{-1}\left(\frac{\Theta}{U}\right), \quad \phi_{p}=\tan ^{-1}\left(\frac{\Gamma}{\Xi}\right),
$$

where

$$
\begin{aligned}
U & =\sqrt{X^{2}+Y^{2}+(Z / 2)^{2}}, \\
\Xi & =-X \cos \theta \cos \phi-Y \sin \phi+\frac{1}{2} Z \sin \theta \cos \phi, \\
\Gamma & =-X \cos \theta \sin \phi+Y \cos \phi+\frac{1}{2} Z \sin \theta \sin \phi, \\
\Theta & =X \sin \theta+\frac{Z}{2} \cos \theta .
\end{aligned}
$$

The first and second derivatives of $\theta_{p}$ and $\phi_{p}$ are

$$
\begin{aligned}
\frac{\partial \theta_{p}}{\partial m_{j}}= & \frac{1}{\left(T^{2}+\Theta^{2}\right) T}\left(\Theta T_{j}^{\prime}-T^{2} \Theta_{j}\right), \\
\frac{\partial^{2} \theta_{p}}{\partial m_{j} \partial m_{k}}= & \frac{1}{\left(T^{2}+\Theta^{2}\right)^{2} T^{3}}\left[T^{2} \Theta\left(T^{2}+\Theta^{2}\right) T_{j k}^{\prime}-\Theta\left(3 T^{2}+\Theta^{2}\right) T_{j}^{\prime} T_{k}^{\prime}\right. \\
& \left.\quad+2 T^{4} \Theta \Theta_{j} \Theta_{k}+T^{2}\left(T^{2}-\Theta^{2}\right)\left(T_{j}^{\prime} \Theta_{k}+T_{k}^{\prime} \Theta_{j}\right)\right], \\
\frac{\partial \phi_{p}}{\partial m_{j}}= & \frac{1}{\Xi^{2}+\Gamma^{2}}\left(\Xi \Gamma_{j}-\Xi_{j} \Gamma\right), \\
\frac{\partial^{2} \phi_{p}}{\partial m_{j} \partial m_{k}}= & \frac{1}{\left(\Xi^{2}+\Gamma^{2}\right)^{2}}\left[\Xi \Gamma\left(\Xi_{j} \Xi_{k}-\Gamma_{j} \Gamma_{k}\right)-\left(\Xi^{2}-\Gamma^{2}\right)\left(\Xi_{j} \Gamma_{k}+\Xi_{k} \Gamma_{j}\right)\right],
\end{aligned}
$$

where

$$
T=\sqrt{U^{2}-\Theta^{2}}=\sqrt{X^{2} \cos ^{2} \theta+Y^{2}+\frac{Z^{2}}{4} \sin ^{2} \theta-X Z \cos \theta \sin \theta}
$$

and $\Xi_{j}, \Xi_{k}, \Gamma_{j}, \Gamma_{k}, \Theta_{j}$ and $\Theta_{k}$ are their derivatives, as

$$
\Xi_{j}=-X_{j} \cos \theta \cos \phi-Y_{j} \sin \phi+\frac{1}{2} Z_{j} \sin \theta \cos \phi
$$

$T_{j}^{\prime}$ and $T_{j k}^{\prime}$ are defined as

$$
\begin{aligned}
T_{j}^{\prime} & =T \frac{\partial T}{\partial m_{j}}=X X_{j} \cos ^{2} \theta+Y Y_{j}+\frac{1}{4} Z Z_{j} \sin ^{2} \theta-\frac{1}{2}\left(X_{j} Z+X Z_{j}\right) \cos \theta \sin \theta, \\
T_{j k}^{\prime} & =\frac{\partial T_{j}^{\prime}}{\partial m_{k}}=X_{j} X_{k} \cos ^{2} \theta+Y_{j} Y_{k}+\frac{1}{4} Z_{j} Z_{k} \sin ^{2} \theta-\frac{1}{2}\left(X_{j} Z_{k}+X_{k} Z_{j}\right) \cos \theta \sin \theta,
\end{aligned}
$$


and $T_{k}^{\prime}$ is similar to $T_{j}^{\prime}$.

The VDM can be calculated as

$$
M=a^{3} U=a^{3} \sqrt{X^{2}+Y^{2}+(Z / 2)^{2}},
$$

To express the VDM in the usual unit of $\mathrm{Am}^{2}$, it is necessary to multiply the right-hand side by a factor of $4 \pi / \mu_{0}=10^{7}$. The first and second derivatives of the elements of VGP are

$$
\begin{aligned}
\frac{\partial M}{\partial m_{j}} & =\frac{a^{3} U_{j}^{\prime}}{U}, \\
\frac{\partial^{2} M}{\partial m_{j} \partial m_{k}} & =\frac{a^{3}}{U^{3}}\left(U U_{j k}^{\prime}-U_{j}^{\prime} U_{k}^{\prime}\right),
\end{aligned}
$$

where $U_{j}^{\prime}, U_{k}^{\prime}$ and $U_{j k}^{\prime}$ are defined similarly to (A.14).

As was the case in paleomagnetic directions, the mean of the VGPs is defined by summing the unit vector in the direction of the individual VGP. Thus, we need the expressions for the direction cosines $\left(\xi_{p}, \eta_{p}, \zeta_{p}\right)$ of the pole:

$$
\xi_{p}=\sin \theta_{p} \cos \phi_{p}=\frac{\Xi}{U}, \quad \eta_{p}=\sin \theta_{p} \sin \phi_{p}=\frac{\Gamma}{U}, \quad \zeta_{p}=\cos \theta_{p}=\frac{\Theta}{U}
$$

where $\Xi$ and $\Gamma$ are defined in (A.10). The first and second derivatives of $\xi_{p}$ can be obtained as

$$
\begin{aligned}
\frac{\partial \xi_{p}}{\partial m_{j}} & =\frac{\Xi_{j}}{U}-\frac{U_{j}^{\prime} \Xi}{U^{3}}, \\
\frac{\partial^{2} \xi_{p}}{\partial m_{j} \partial m_{k}} & =\frac{3 U_{j}^{\prime} U_{k}^{\prime} \Xi}{U^{5}}-\frac{U_{j k}^{\prime} \Xi+U_{j}^{\prime} \Xi_{k}+U_{k}^{\prime} \Xi_{j}}{U^{3}} .
\end{aligned}
$$

The derivatives of $\eta_{p}$ and $\zeta_{p}$ can be obtained by changing the terms concerned with the numerators of Eq. (A.17).

\section{A.4 Mean directions defined by Fisher's method}

The Fisherian means of nonlinear data can be expanded following the general method given by Eq. (19). Specifically, we treat the nonlinear quantities $\bar{x}_{F}, \bar{y}_{F}, \bar{z}_{F}, \bar{D}_{F}$, and $\bar{I}_{F}$ as functions of $E_{x}, E_{y}$, and $E_{z}$. For instance, for the Fisherian mean of $x$ we have

$$
\bar{x}_{F}=x_{0}+\frac{1}{2}\left(\frac{\partial \bar{x}_{F}}{\partial E_{x}} \Delta E_{x}+\frac{\partial \bar{x}_{F}}{\partial E_{x}} \Delta E_{y}+\frac{\partial \bar{x}_{F}}{\partial E_{x}} \Delta E_{z}\right)_{0}+\cdots
$$

As usual, the suffix 0 indicates that the values are to be evaluated for the reference model $\boldsymbol{m}_{0}$. The partial derivatives can be calculated from (12),

$$
\frac{\partial \bar{x}_{F}}{\partial E_{x}}=\frac{1}{E_{f}}-\frac{E_{x}^{2}}{E_{f}^{3}}, \quad \frac{\partial \bar{x}_{F}}{\partial E_{y}}=-\frac{E_{x} E_{y}}{E_{f}^{3}}, \quad \frac{\partial \bar{x}_{F}}{\partial E_{z}}=-\frac{E_{x} E_{z}}{E_{f}^{3}} .
$$

On the other hand, the deviations of the means direction cosines are given by (9)

$$
\Delta E_{x}=\frac{1}{2} \sum_{j} \frac{\partial^{2} x}{\partial m_{j}^{2}} \sigma_{j}^{2}+\cdots, \quad \text { etc. }
$$

Thus, the expression of $\bar{x}_{F}$ accurate to second order $\left(\sigma_{j}^{2}\right)$ is

$$
\bar{x}_{F}=x_{0}+\frac{1}{2} \sum_{j}\left[\frac{\partial^{2} x}{\partial m_{j}^{2}}-\frac{X}{F^{2}}\left(X \frac{\partial^{2} x}{\partial m_{j}^{2}}+Y \frac{\partial^{2} y}{\partial m_{j}^{2}}+Z \frac{\partial^{2} z}{\partial m_{j}^{2}}\right)\right]_{0} \sigma_{j}^{2}+\mathcal{O}\left(\sigma_{j}^{4}\right) .
$$

It can be shown from (A.8) that

$$
\begin{gathered}
X \frac{\partial^{2} x}{\partial m_{j}^{2}}+Y \frac{\partial^{2} y}{\partial m_{j}^{2}}+Z \frac{\partial^{2} z}{\partial m_{j}^{2}}=-\frac{G_{j j}}{F}+\frac{G_{j}^{2}}{F^{3}} \\
\frac{\partial^{2} x}{\partial m_{j}^{2}}-\frac{X}{F^{2}}\left(X \frac{\partial^{2} x}{\partial m_{j}^{2}}+Y \frac{\partial^{2} y}{\partial m_{j}^{2}}+Z \frac{\partial^{2} z}{\partial m_{j}^{2}}\right)=-\frac{2 X_{j} G_{j}}{F^{3}}+\frac{2 X G_{j}^{2}}{F^{5}}
\end{gathered}
$$

The final forms of the Fisher-mean direction cosines and directions, accurate to second order, are

$$
\bar{x}_{F}=x_{0}+\sum_{j}\left[\frac{X G_{j}^{2}}{F^{5}}-\frac{X G_{j}}{F^{3}}\right]_{0} \sigma_{j}^{2}+\mathcal{O}\left(\sigma_{j}^{4}\right),
$$




$$
\begin{aligned}
& \bar{y}_{F}=y_{0}+\sum_{j}\left[\frac{Y G_{j}^{2}}{F^{5}}-\frac{Y_{j} G_{j}}{F^{3}}\right]_{0} \sigma_{j}^{2}+\mathcal{O}\left(\sigma_{j}^{4}\right), \\
& \bar{z}_{F}=z_{0}+\sum_{j}\left[\frac{Z G_{j}^{2}}{F^{5}}-\frac{Z_{j} G_{j}}{F^{3}}\right]_{0} \sigma_{j}^{2}+\mathcal{O}\left(\sigma_{j}^{4}\right), \\
& \bar{D}_{F}=D_{0}+\sum_{j}\left[\frac{\left(X_{j} Y-X Y_{j}\right) G_{j}}{F^{2} H^{2}}\right]_{0} \sigma_{j}^{2}+\mathcal{O}\left(\sigma_{j}^{4}\right), \\
& \bar{I}_{F}=I_{0}+\sum_{j}\left[\frac{G_{j}\left(Z\left(X X_{j}+Y Y_{j}\right)-H^{2} Z_{j}\right)}{F^{4} H}\right]_{0} \sigma_{j}^{2}+\mathcal{O}\left(\sigma_{j}^{4}\right) .
\end{aligned}
$$

\section{A.5 Derivatives with respect to the linear components $X, Y$ and $Z$}

In order to consider only the effect of experimental errors, we can fix the field model to the reference model $\boldsymbol{m}_{0}$, which means that there is no contribution from the fluctuation of the field model itself. In this case, the observed linear elements can be expressed as $X=X_{0}+\Delta X$, etc., where $X_{0}$ is the value corresponding to the field model and $\Delta X$ is the error in measurement. Thus, we can treat the three orthogonal components as independent variables and proceed to estimate the effect of errors by taking the derivatives of the field elements with respect to $X, Y$, and $Z$ as we did in (1).

The first and second derivatives of direction cosines $x$ can be obtained as

$$
\frac{\partial x}{\partial X}=\frac{1}{F^{3}}\left(F^{2}-X^{2}\right), \quad \frac{\partial x}{\partial Y}=-\frac{X Y}{F^{3}}, \quad \frac{\partial x}{\partial Z}=-\frac{X Z}{F^{3}}
$$

and

$$
\begin{aligned}
\frac{\partial^{2} x}{\partial X^{2}} & =-\frac{3 X}{F^{5}}\left(F^{2}-X^{2}\right), \quad \frac{\partial^{2} x}{\partial Y^{2}}=\frac{X}{F^{5}}\left(3 Y^{2}-F^{2}\right), \quad \frac{\partial^{2} x}{\partial Z^{2}}=\frac{X}{F^{5}}\left(3 Z^{2}-F^{2}\right), \\
\frac{\partial^{2} x}{\partial X \partial Y} & =\frac{\partial^{2} x}{\partial Y \partial X}=\frac{Y}{F^{5}}\left(2 X^{2}-Y^{2}-Z^{2}\right), \\
\frac{\partial^{2} x}{\partial X \partial Z} & =\frac{\partial^{2} x}{\partial Z \partial X}=\frac{Z}{F^{5}}\left(2 X^{2}-Y^{2}-Z^{2}\right), \\
\frac{\partial^{2} x}{\partial Y \partial Z} & =\frac{\partial^{2} x}{\partial Z \partial Y}=\frac{3 X Y Z}{F^{5}} .
\end{aligned}
$$

The derivatives of other direction cosines $(y$ and $z$ ) can be obtained by cyclically replacing $X, Y, Z$ in the above expressions. 